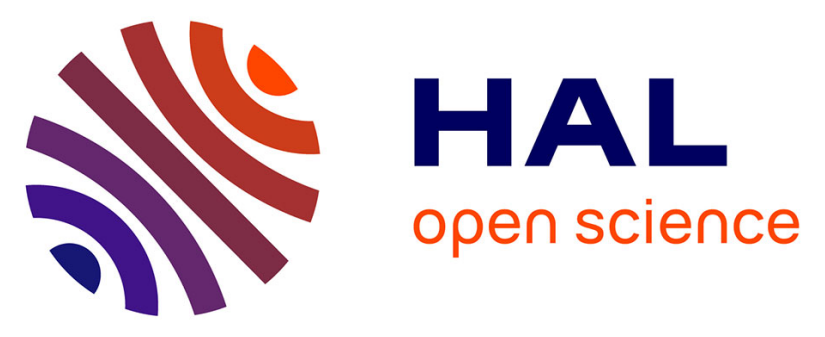

\title{
Surface-based electrophysiology modeling and assessment of physiological simulations in atria
}

Annabelle Collin, Jean-Frédéric Gerbeau, Mélèze Hocini, Michel Haïssaguerre, Dominique Chapelle

\section{- To cite this version:}

Annabelle Collin, Jean-Frédéric Gerbeau, Mélèze Hocini, Michel Haïssaguerre, Dominique Chapelle. Surface-based electrophysiology modeling and assessment of physiological simulations in atria. FIMH - 7th International Conference on Functional Imaging and Modeling of the Heart, Jun 2013, Londres, United Kingdom. pp.352-359, 10.1007/978-3-642-38899-6_42 . hal-00815974

\author{
HAL Id: hal-00815974 \\ https://hal.inria.fr/hal-00815974
}

Submitted on 19 Apr 2013

HAL is a multi-disciplinary open access archive for the deposit and dissemination of scientific research documents, whether they are published or not. The documents may come from teaching and research institutions in France or abroad, or from public or private research centers.
L'archive ouverte pluridisciplinaire HAL, est destinée au dépôt et à la diffusion de documents scientifiques de niveau recherche, publiés ou non, émanant des établissements d'enseignement et de recherche français ou étrangers, des laboratoires publics ou privés. 


\title{
Surface-based electrophysiology modeling and assessment of physiological simulations in atria
}

\author{
A. Collin ${ }^{1}$, J.-F. Gerbeau ${ }^{2}$, M. Hocini ${ }^{3}$, M. Haïssaguerre ${ }^{3}$ and D. Chapelle ${ }^{1}$ \\ 1 Inria Saclay Ile-de-France, M $\exists D I S I M$ Team, Palaiseau, France \\ annabelle.collin@inria.fr \\ 2 Inria Paris-Rocquencourt, REO Team, Le Chesnay, France \\ 3 Hôpital Cardiologique du Haut-Lévêque and Institut LIRYC, \\ Bordeaux-Pessac, France
}

\begin{abstract}
The objective of this paper is to assess a previously-proposed surface-based electrophysiology model with detailed atrial simulations. This model - derived and substantiated by mathematical arguments is specifically designed to address thin structures such as atria, and to take into account strong anisotropy effects related to fiber directions with possibly rapid variations across the wall thickness. The simulation results are in excellent adequacy with previous studies, and confirm the importance of anisotropy effects and variations thereof. Furthermore, this surface-based model provides dramatic computational benefits over 3D models with preserved accuracy.
\end{abstract}

\section{Introduction}

There is a very important medical need for modeling the electrical activity of the heart in general, and in the atria in particular, e.g. with a view to therapy planning assistance in radiofrequency ablation for patients suffering from atrial fibrillation [10]. In addition to generic difficulties inherent to electrophysiology modeling, namely, modeling complexity and computational intensiveness, atria modeling features specific difficulties, in particular due to their very thin walls - mostly apparent as thick surfaces in medical imaging - which requires much refined meshes. Moreover, there is a major challenge in taking into account the anisotropy resulting from the preferred conduction direction along the muscle fibers, which is also known to vary extremely rapidly across the wall thickness [7].

The electrical wave propagating in the cardiac tissue can be represented by a nonlinear reaction-diffusion partial differential equation (PDE), coupled with ordinary differential equations (ODEs) representing cellular activity. In this study, we consider the $2 \mathrm{D}$ - namely, surface-based - model proposed and mathematically substantiated in [1], derived from the bidomain model (see for example [1113]), and defined over the midsurface of the thin region. This surface-based model was specifically designed for thin cardiac structures - the atrial walls, in particular - and takes into account the strong anisotropy variations across the thickness. This model was already successfully numerically assessed in [1] by comparing the resulting simulations with reference 3D simulations on thin domains of simple 
geometries, with dramatic benefits in computation times. Our objective here is to further assess this model with physiological simulations of the atrial electrophysiology.

\section{Model}

\subsection{Atrial mesh}

We produced a surface mesh representing the mid-surface of the two atria. Starting from the Zygote ${ }^{4}$ heart model - a geometric model based on actual anatomical data - we used the 3 -matic ${ }^{5}$ software to obtain a computationally-correct surface mesh, and the Yams [5] meshing software to further process and refine the surface mesh. Figure 1 shows the posterior and anterior views of the mesh, which contains about 26,000 triangles and 13,500 vertices. The anatomy corresponds to ventricular end-systole, namely, when the atrial chamber has its greatest internal volume. This anatomical surface was compared with literature reports of normal human atrial dimension indicators, see for example $[2,8]$, and found to be within standard ranges, see Table 1 .

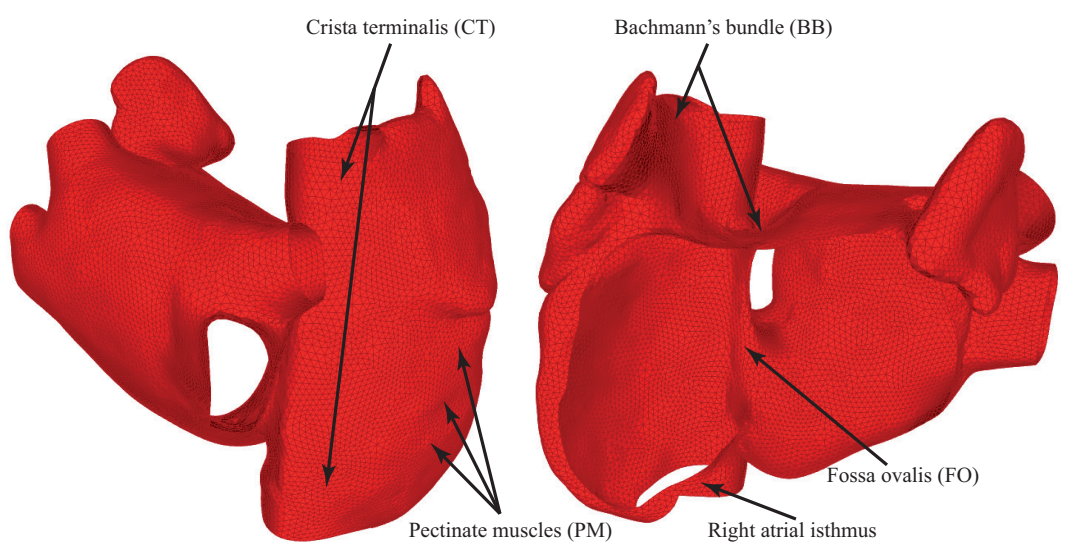

Fig. 1. Atrial mesh posterior (left) and anterior (right) views, with main specific regions

\section{$2.2 \quad$ Fibers}

Cardiac tissue has a fiber architecture. As the electrical conductivity is higher along than across the fiber direction, fiber orientation is very important in electric activation propagation. The specificities of the atria are that the walls are

\footnotetext{
${ }^{4}$ www.3dscience.com

${ }^{5}$ www.materialise.com
} 


\begin{tabular}{|c|c|c|}
\hline LA indicators at ventricular end-systole & Normal (from $[2,8]$ ) & LA model dimensions \\
\hline diameter $(\mathrm{cm})$ & $2.8-4.0$ & 3.0 \\
major axis $(\mathrm{cm})$ & $4.1-6.1$ & 4.65 \\
area $\left(\mathrm{cm}^{2}\right)$ & $15.0-20.0$ & 15.9 \\
volume $(\mathrm{ml})$ & $41-75$ & 47 \\
diameters of pulmonary veins $(\mathrm{cm})$ & $1.1-1.5$ & $1.14-1.45$ \\
\hline
\end{tabular}

Table 1. Assessment of left atrial dimension indicators

very thin, and that fibers orientations may vary extremely rapidly across the thickness. We use $[7,9]$ to identify and prescribe the fibers directions at the endocardium and epicardium, see Figure 2. This figure also displays the angle $\theta$, defined as half of the angular variation between the endocardium and the epicardium.

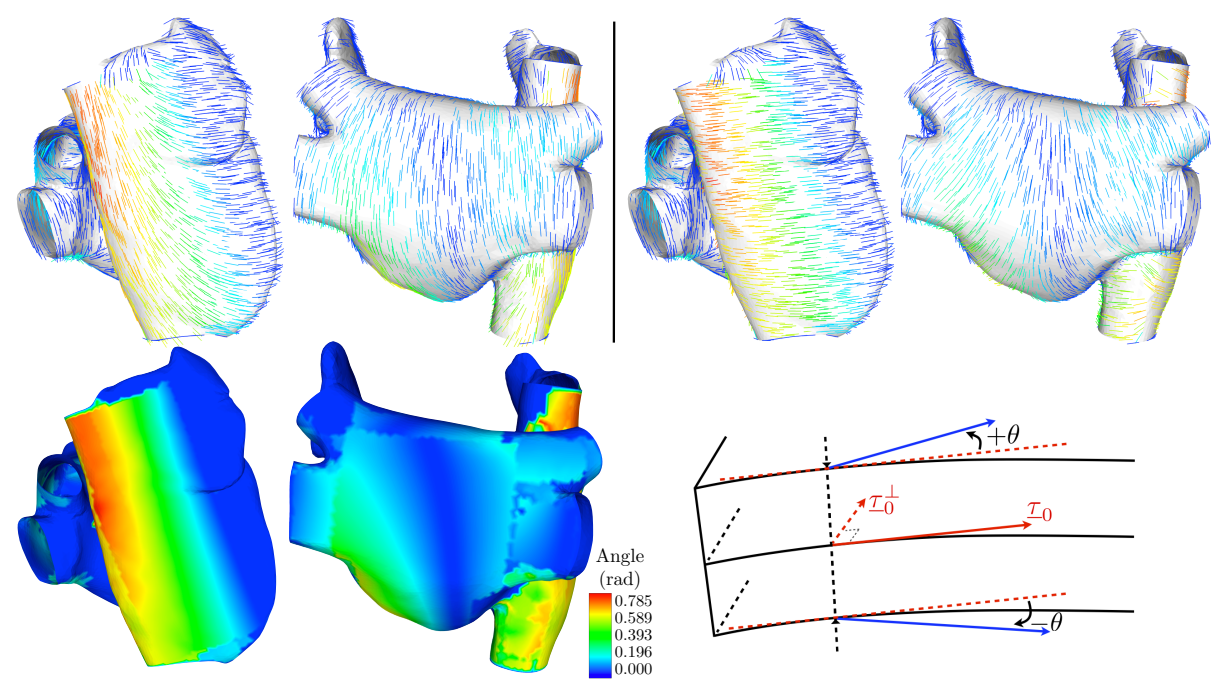

Fig. 2. Fibers directions at the epicardium (top-left) and endocardium (top-right), angle $\theta$ (bottom-left), and angular variation visualization (bottom-right)

\subsection{Surface-based bidomain model}

We denote by $\mathcal{S}$ the midsurface of the wall. The variational bidomain surface model that we propose can be written in terms of the extracellular potential $u_{e}$, the transmembrane potential $V_{m}=u_{i}-u_{e}$, with $u_{i}$ the intracellular potential, 
as follows [1]. Find $\left(V_{m}, u_{e}\right)$ with $\int_{\mathcal{S}} u_{e} d S=0$, such that for all $t>0$,

$$
\left\{\begin{array}{r}
A_{m} \int_{\mathcal{S}}\left(C_{m} \frac{\partial V_{m}}{\partial t}+I_{i o n}\left(V_{m}\right)\right) \phi d S+\int_{\mathcal{S}}\left(\underline{\underline{\sigma}}_{i} \cdot\left(\underline{\nabla} V_{m}+\underline{\nabla} u_{e}\right)\right) \cdot \underline{\nabla} \phi d S \\
=A_{m} \int_{\mathcal{S}} I_{a p p} \phi d S, \quad \forall \phi \\
\int_{\mathcal{S}}\left(\left(\underline{\underline{\sigma}}_{i}+\underline{\underline{\sigma}}_{e}\right) \cdot \underline{\nabla} u_{e}\right) \cdot \underline{\nabla} \psi d S+\int_{\mathcal{S}}\left(\underline{\underline{\sigma}}_{i} \cdot \underline{\nabla} V_{m}\right) \cdot \underline{\nabla} \psi d S=0, \quad \forall \psi
\end{array}\right.
$$

also with $\int_{\mathcal{S}} \psi d S=0$, and where $A_{m}$ is a positive constant denoting the ratio of membrane area per unit volume, $C_{m}$ the membrane capacitance per unit surface, $I_{i o n}\left(V_{m}\right)$ a reaction term representing the ionic current across the membrane and also depending on local ionic variables satisfying additional ODEs, and $I_{a p p}$ a given applied stimulus current.

We define the intra- and extra-cellular diffusion tensors $\underline{\underline{\sigma}}_{i}$ and $\underline{\underline{\sigma}}_{e}$ by

$$
\underline{\underline{\sigma}}_{i, e}=\sigma_{i, e}^{t} \underline{\underline{I}}+\left(\sigma_{i, e}^{l}-\sigma_{i, e}^{t}\right)\left[I_{0}(\theta) \underline{\tau}_{0} \otimes \underline{\tau}_{0}+J_{0}(\theta) \underline{\tau}_{0}^{\perp} \otimes \underline{\tau}_{0}^{\perp}\right]
$$

where $\underline{I}$ denotes the identity tensor in the tangential plane - also sometimes called the surface metric tensor $-\underline{\tau}_{0}$ is a unit vector parallel to the local fiber direction on the atria midsurface, and $\underline{\tau}_{0}^{\perp}$ such that $\left(\underline{\tau}_{0}, \underline{\tau}_{0}^{\perp}\right)$ gives an orthonormal basis of the tangential plane. The effect of angular variations enters the model through the coefficients $I_{0}(\theta)=\frac{1}{2}+\frac{1}{4 \theta} \sin (2 \theta)$ and $J_{0}(\theta)=1-I_{0}(\theta)$. Note that $J_{0}(\theta)=0$ (and $I_{0}(\theta)=1$ ) if and only if $\theta=0$ - namely, constant direction across the thickness - and then $\underline{\underline{\sigma}}_{i, e}=\sigma_{i, e}^{t} \underline{\underline{I}}+\left(\sigma_{i, e}^{l}-\sigma_{i, e}^{t}\right) \underline{\tau}_{0} \otimes \underline{\tau}_{0}$ as expected for a single fiber direction. By contrast, angular variations make $I_{0}$ decrease and $J_{0}$ increase in (2), which renders diffusion "more isotropic". This model derived from a detailed asymptotic analysis thus allows to take into account the rapid variations of the fiber direction.

The current $I_{i o n}$ can be described by a physiological or a phenomenological model. In this study, the physiological model proposed by Courtemanche, Ramirez and Nattel in [3] - most widely accepted for atria modeling - is considered.

The values of the membrane parameters are $A_{m}=200.0 \mathrm{~cm}^{-1}$ and $C_{m}=$ $10^{-3} \mathrm{mF} . \mathrm{cm}^{-2}$. The values of the conductivity parameters vary substantially depending on the specific areas considered and are given in Table $2\left(\right.$ all in S.cm ${ }^{-1}$ ). The Bachmann bundle (BB), the Crista Terminalis (CT) and the pectinate muscles (PM) are regions of established fast conduction. By contrast, the Fossa Ovalis (FO) and the Isthmus of the right atrial floor (IRA) are regions of known slow conduction. All these specific regions are depicted in Figure 1.

The two atria are connected via two regions only - in the mesh connectivity, hence also in terms of electrical conduction - namely, the Bachmann bundle and the Fossa Ovalis. 


\begin{tabular}{|c|c|c|c|c|c|c|c|}
\hline & regular tissue & $\mathrm{PM}$ & $\mathrm{CT}$ & $\mathrm{BB}$ & $\mathrm{IRA}$ & $\mathrm{FO}$ & $\mathrm{SN}$ \\
\hline$\sigma_{i}^{t}$ & $2.210^{-4}$ & $3.310^{-4}$ & $6.610^{-4}$ & $1.04510^{-3}$ & $8.25010^{-5}$ & $2.210^{-4}$ & $2.210^{-4}$ \\
\hline$\sigma_{i}^{l}$ & $2.210^{-3}$ & $3.89410^{-3}$ & $9.57010^{-3}$ & $1.63910^{-2}$ & $9.73510^{-4}$ & $2.00210^{-3}$ & $2.00210^{-3}$ \\
\hline$\sigma_{e}^{t}$ & $9.010^{-4}$ & $1.35010^{-3}$ & $2.710^{-3}$ & $4.27510^{-3}$ & $3.37510^{-4}$ & $9.010^{-4}$ & $9.010^{-4}$ \\
\hline$\sigma_{e}^{l}$ & $2.210^{-3}$ & $3.69010^{-3}$ & $8.55010^{-3}$ & $1.43510^{-2}$ & $9.22510^{-4}$ & $2.07010^{-3}$ & $2.07010^{-3}$ \\
\hline
\end{tabular}

Table 2. Conductivity parameters
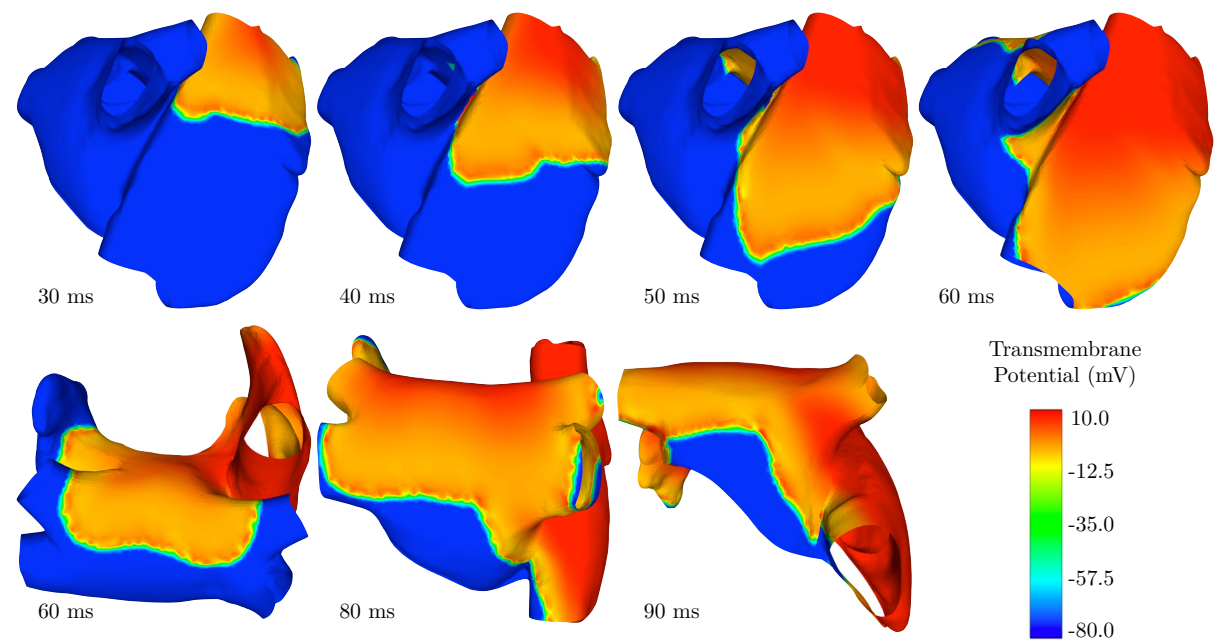

Transmembrane Potential (mV)

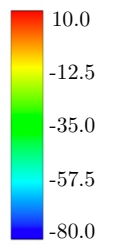

Fig. 3. Simulation of atrial depolarization
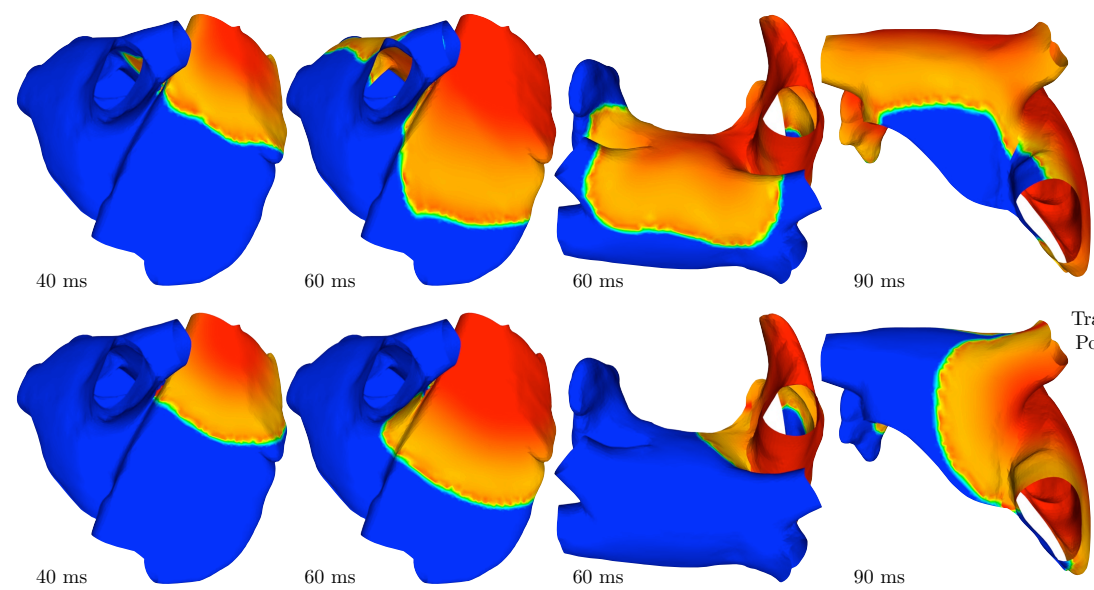

$90 \mathrm{~ms}$

$60 \mathrm{~ms}$

$60 \mathrm{~ms}$

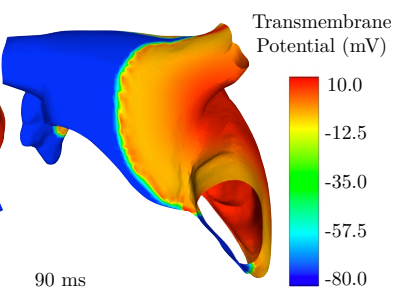

Fig. 4. Simulations of atrial depolarization for homogeneous case i.e $\theta=0$ (top) and for homogeneous and isotropic case (bottom) 

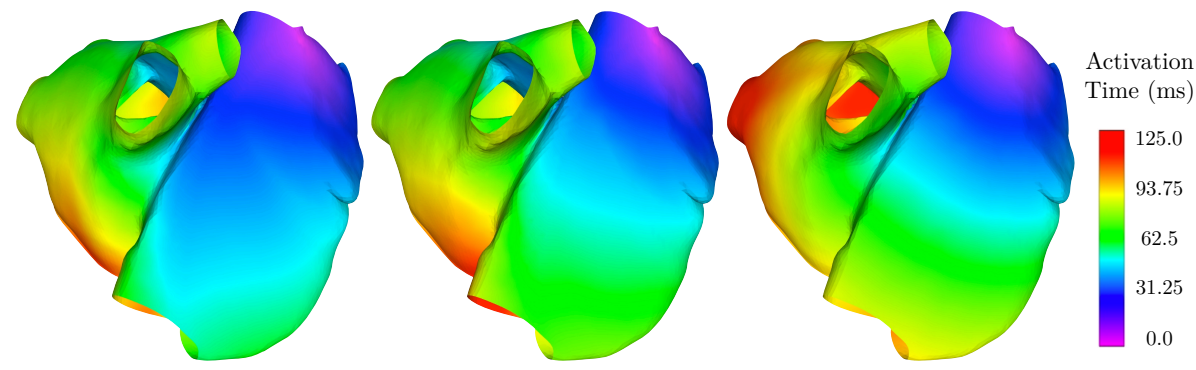

Fig. 5. Activation time maps for the three cases: anisotropic-heterogeneous (left), anisotropic-homogeneous (middle) and isotropic-homogeneous (right)

\section{Simulations results}

Figure 3 displays the simulation results obtained with the above-described model. Activation is initiated at the sinus node with a stimulus of $2 \mathrm{~ms}$ in duration and sufficient strength to cause the initiation of a propagating wavefront. We compare our results to several 3D modeling studies $[9,6,4]$.

As observed in the figure, by $30 \mathrm{~ms}$ the wave quickly spreads along the Crista Terminalis as a consequence of the high conductivity in this part. Importantly, the depolarizing wave has now traversed the Bachmann bundle and the first activation of the left atrium has occurred at $29.6 \mathrm{~ms}$. This compares very well with the findings of the modeling study [6] giving the left atrium activation at $29.7 \mathrm{~ms}$. At $t=40 \mathrm{~ms}$, we clearly see the effect of the anisotropy of the Crista Terminalis, and of the pectinate muscles already observed in [9], as the wavefront becomes nearly triangular - as also seen in [6]. The wavefront has encircled the superior Vena Cava. The Fossa Ovalis, which is the second atrial connection, becomes active $(42.8 \mathrm{~ms})$. By $50 \mathrm{~ms}$, because of the rapid conduction in the Bachmann bundle, the wave spreads to the left atrial appendage and has activated a substantial part of the left atrial wall. The Fossa Ovalis is very active. The activation of the right atrial appendage is complete. At $t=60 \mathrm{~ms}$, in the right atrium the floor is the last part that remains unexcited. The wave has encircled the mouth of the left atrial appendage at $72.8 \mathrm{~ms}$. By $t=80 \mathrm{~ms}$, the only part that remains unaffected by the wave in the right atrium is the isthmus, because of the reduced conductivity there. In the left atrium, at $t=90 \mathrm{~ms}$ we can see three separate wavefronts, also obtained in [6,4]. The depolarization of the left atrial appendage is complete at $95.8 \mathrm{~ms}$. At $100 \mathrm{~ms}$, only a small part of the left atrium in the shape of a parallelepiped is still inactive. The depolarization of the right and left atrium are complete at $101.8 \mathrm{~ms}$ and $109.6 \mathrm{~ms}$, respectively. This is in good adequacy with the timings found in [6], namely, $99.3 \mathrm{~ms}$ and $108.2 \mathrm{~ms}$, respectively, while [9] gives $115.0 \mathrm{~ms}$ for the left atrium, and [4] $119.0 \mathrm{~ms}$. 


\section{Discussion}

For comparison purposes, we also display in Figure 4-top simulation results obtained when disregarding the fiber direction variations across the thickness, namely, when taking $\theta=0$. We observe major differences with the abovediscussed physiological simulation. In particular, the anisotropy effects characteristic of the Crista Terminalis are much less clearly seen, and the triangular shape is hardly observed, indeed. Overall, the activation timings are longer, in particular due to slower propagation in the Crista Terminalis, with complete depolarization occurring as late as $116.2 \mathrm{~ms}$ and $115.4 \mathrm{~ms}$ for the right and left atria, respectively. The last results displayed in Figure 4-bottom correspond to an homogeneous and isotropic tissue throughout. We increased the homogeneous conductivity with $\sigma_{i}=8.010^{-4} \mathrm{~S} . c m^{-1}$ and $\sigma_{e}=3.110^{-3} \mathrm{~S} . c m^{-1}$ to obtain roughly comparable depolarization timings. The resulting activation profile, however, is totally unrealistic - as already noted in [9] - see Figure 5 displaying the activation time maps for the three cases analyzed.

\section{Concluding remarks}

We have presented detailed simulation results of a surface-based electrophysiology bidomain model applied with an anatomical model of the atria. The results obtained are in excellent adequacy with previous studies, and confirm the importance of fiber-related anisotropy effects and of their strong variations across the wall thickness. Furthermore, this surface-based model provides dramatic computational benefits over 3D models with preserved accuracy [1]. Further perspectives include applications to radio-frequency ablation planning assistance, for which computational effectiveness - less than 3 minutes for a complete simulation on a standard workstation - will be of great value.

\section{References}

1. D. Chapelle, A. Collin, and J.-F. Gerbeau. A surface-based electrophysiology model relying on asymptotic analysis and motivated by cardiac atria modeling. M3AS, 2012. In press, http://hal.inria.fr/hal-00723691/en.

2. G.I. Cohen, M. White, R.A. Sochowski, A.Z. Klein, P.D. Bridge, W.J. Steward, and K.L. Chang. Reference values for normal adult transesophageal echocardiographic measurements. Journal of the American Society of Echocardiography, (8):221-230, 1995.

3. M. Courtemanche, R.J. Ramirez, and S. Nattel. Modeling atrial fiber orientation in patient-specific geometries: A semi-automatic rule-based approach. American Journal of Physiology, (275):H301-H321, 1998.

4. D. Deng, Y. Gong, G. Shou, and P. Jiao. Simulation of biatrial conduction via different pathways during sinus rhythm with a detailed human atrial model. Journal of Zheijiang University-SCIENCE B (Biomedicine and Biotechnology), pages 1862-1783, 2012. 
5. P. Frey. Yams: A fully automatic adaptive isotropic surface remeshing procedure. Technical report 0252, Inria, Rocquencourt, France, Nov. 2001.

6. D.M. Harrild and S.H. Craig. A computer model of normal conduction in the human atria. Circulation Research, (87):e25-e36, 2000.

7. S.Y. Ho, R.H. Anderson, and D. Sánchez-Quintana. Atrial structure and fibres: morphologic bases of atrial conduction. Cardiovascular Research, (54):325-336, 2002.

8. P. Jiamsripong, T. Honda, C.S. Reuss, R.T. Hurst, H.P. Chaliki, D.E. Grill, S.L. Schneck, R. Tyler, B.K. Khandheria, and S.J. Lester. Three methods for evaluation of left atrial volume. European Journal of Echocardiography, (9):351-355, 2008.

9. M. Krueger, V. Schmidt, C. Tobón, F. Weber, C. Lorenz, D. Keller, H. Barschdorf, M. Burdumy, P. Neher, G. Plank, K. Rhode, G. Seemann, D. Sánchez-Quintana, J. Saiz, R. Razavi, and O. Dössel. Modeling atrial fiber orientation in patientspecific geometries: a semi-automatic rule-based approach. Functional Imaging and Modeling of the Heart, pages 223-232, 2011.

10. S. Matsuo et al. Clinical predictors of termination and clinical outcome of catheter ablation for persistent atrial fibrillation. Journal of the American College of Cardiology, 54(9):788-795, 2009.

11. A.J. Pullan, M.L. Buist, and L.K. Cheng. Mathematically Modeling the Electrical Activity of the Heart. World Scientific, 2005.

12. F.B. Sachse. Computational Cardiology: Modeling of Anatomy, Electrophysiology and Mechanics. Springer-Verlag, 2004.

13. J. Sundnes, G.T. Lines, X. Cai, B.F. Nielsen, K.A. Mardal, and A. Tveito. Computing the Electrical Activity in the Heart, volume 1 of Monographs in Computational Science and Engineering. Springer-Verlag, 2006. 